\title{
Improving construction duration forecasts and management of construction operations
}

\author{
Aldyn-kys Dadar ${ }^{1, *}$, Sergey Bolotin ${ }^{2}$, Aslan Malsagov ${ }^{2}$, and Zita Oolakai ${ }^{1}$ \\ ${ }^{1}$ Tuvan State University, Lenina street, 36, Kyzyl, 667000, Russia \\ ${ }^{2}$ St. Petersburg State University of Architecture and Civil Engineering, 2-nd Krasnoarmeiskaja street, \\ 4, St.Petersburg, 190005, Russia
}

\begin{abstract}
To address the relevant construction challenges, it is necessary both to improve the existing construction duration forecasting methods and to develop optimal construction management methods. That being said, effective implementation of general management functions is defined by information interaction between management subjects and objects as a system bringing together construction management stakeholders. Very often the construction practice reveals that some contractors' statistics related to execution of works can altogether be absent or be unrepresentative. With this fact borne in mind, the authors of this material focus on using nonparametric statistic or distribution-free methods. On the other hand, the lack of representative statistical data indirectly indicates to ineffective management of the construction process. The article shows that the possible solution of the construction duration forecasting task is the methodology of neural and statistical simulation. We suggest that the efficiency of expeditious construction management should be assessed by calculating the information entropy, while regulatory action aimed at increasing managerial energy must be achieved by optimally allocating costs among the various elements oriented towards resource-based management. The presented model of improving construction duration forecasts allows the optimization of construction regulation process, which must increase its organizational and technological reliability.
\end{abstract}

\section{Introduction}

Construction as the process of creating an immovable object is defined by general management functions, most important of them are planning, organization, monitoring and regulation. In their turn, each of these functions is polymorphic, i.e. each function can be represented by a multitude of various forms. Let's define the forms of general management functions for our proposed study. The planning function is first of all about developing a construction schedule and forecasting the construction duration. The organization function defines the distribution of white-collar and blue-collar labor in implementing a plan of construction operations. The monitoring function is about updating the construction

*Corresponding author: daryi@mail.ru 
activities executed as of a certain date. The regulation function is related to the impact upon a managed facility (object) via changes in the planning, organization and management of the works done. Under the organization of construction operations, we understand a totality of processes or activities that lead to the formation and improvement of linkages in the system of primary management of the construction process [1].

Because construction operations are a process, general management functions are constantly performed via discreet intervals. Whenever construction operations are organized as a system, the latter is divided into management objects and subjects. There are direct and inverse linkages at play between the distinguished elements, these linkages characterizing the information integrity of the general construction management functions. For all that, it is unequivocally believed that technological processes [2] are the object of managing construction operations. If we were to classify construction management subjects by positions, it should be noted that there are certain terminological differences in different countries. For example, in the U.S., there is no uniformity like "the table of ranks" adopted in the Russian Federation: in different U.S. companies, a lower-tier steward can be named either a section engineer or foreman which correspond with such positions in Russia as senior worker, team leader or taskmaster in Russia [3]. Yet it is characteristic of the task under review that any list of duties performed by a lower-tier manager includes general management charges. One example is the duties normally executed by a foreman [3]:

- supervision of workers involved in construction works;

- reading blueprints, drawings and specifications;

- appointing to work positions and briefing the workers;

- instructions in how to use various tools and equipment;

- obtaining materials and checking their quality;

- maintaining the documents fixing the course of works;

- bearing responsibility for the volume, quality and safety of works.

All construction operations have scheduled (planned) beginning and ending dates, so to reach the planned performance indicators, it is necessary to ensure constant monitoring of the works' execution and on this basis, following some chosen methods, the date of construction completion is forecast. In most cases there is a gap between the planned and forecast dates, so a subject wields a regulatory impact upon the object under management to close this gap.

The Russian construction practice knows specialized organizations professionally monitoring the works done by contractors. In particular, SetlCity which organized the monitoring of contractors and subcontractors carrying out the construction of residential projects [4], hired a special company to conduct system monitoring [5]. Thus, as a result of verification conducted in January 2017, it was documented that a number of works had been done in the lack of essential organizational and engineering documentation. It was established that those contractors failed to keep within the schedule and ensure decent quality. That being said, the data resulting from this monitoring do not make it possible to draw a quantitative conclusion about the construction delay because of insufficient organizational-engineering (technological) documentation.

The construction practice shows that for some contractors the statistics related to the schedule of works can be absent or be unrepresentative. Proceeding from this fact, we believe that using nonparametric statistic methods would be expedient.

One alternative of nonparametric statistic methods is assuming the duration of works as a random value, with a beta-distribution used as an underlying premise to describe it. This conclusion is indirectly corroborated by the following quote from the article [6]. "Almost in all network-based scheduling systems, it is assumed that the density of temporal distribution of works has three properties: a) continuity, b) unimodality, c) two nonnegative 
points of intersection of this density with the axis of abscises. The simplest distribution with such properties is the beta distribution which can normally be postulated in practice.

Unlike the above-stated axiom, the article [7] suggests that an exponential distribution would be more preferable for statistical simulation of calendar schedules. The authors believe that it occurs whenever the force of regulatory impact is proportionate to the possible deviation of the works' duration from the schedule. It is symptomatic that the article [7] suggests that the exponential distribution should be regarded as optimistic, uniform distribution - as pessimistic, and the triangular distribution - as the most probable of all. Then the following formula is proposed to describe the averaged function of the distribution density of the works' random duration:

$$
f(t)=\frac{1}{(b-a)}\left\{z_{1}+2 z_{2} \frac{(b-t)}{(b-a)}+4 z_{3} \exp \left[-\frac{4(t-a)}{b-a}\right]\right\} .
$$

where $\quad a$-scheduled (optimistic) duration of the work,

$b$ - pessimistic duration of the work found by adding four standard deviations to the planned duration,

$z_{1}, z_{2}, z_{3}$, - weighting factors establishing the normalized weights of presented distributions.

The task of the weights' distribution is usually solved using one of the expert evaluation methods [8], and in this case, using the distribution function described in formula (1) takes the task of statistical modeling to the class of nonparametric statistics problems. So we can make another case for advisability of applying nonparametric statistic methods to solving the tasks of construction operations related to the forecasting of certain works' execution.

In academic literature, you can find those classification hallmarks which predetermine the choice of nonparametric statistic methods [9]. "One of the factors restricting the use of criteria based on the normality assumption is the sample size. As long as the sample is big enough (e.g. 100 or more observations), the random distribution can be thought normal, even if you are not sure that the distribution of the variable in a population is normal. Nevertheless, if the sample is small, the same criteria should be used only if you are certain that the variable indeed has a normal distribution. However, there is no way to check this assumption using a smaller sample." Therefore, the task of improving the existing methods of construction duration forecast and the task of developing new methods geared at effective regulation of the construction process can be thought relevant.

\section{Materials and methods}

Forecasting the duration based on neural simulation methodology. As was stated above, the task of forecasting the durations of execution of certain works is addressed by using some or other distribution functions. Yet the task of forecasting the general end of construction works is even more challenging, since it ought to be solved using a method of statistical simulation of the general works schedule. Let's consider its possible solution based on the neural simulation methodology that was suggested more than 20 years ago [10]. In this type of simulation, one can use Rosenblatt perceptron, because its rather simple scheme allows the formation of the following analytical model:

$$
Y=\frac{1}{1+\operatorname{Exp}\left(\sum_{i}-w_{i} x_{i}\right)},
$$


where $Y$ is the function representing the untimely construction;

$x_{i}$ - independent arguments representing the updated values of underperforming of certain works;

$w_{i}$ - weighting factors found in the course of the system training based on its statistical modeling.

Solving the problem of total duration forecasting based on neural simulation gets down to finding such weighting factors which minimize the deviations of forecast untimeliness values from the respective values figured in the course of statistical simulation. The forecasting procedure in this case is algorithmically split into sequential execution of the following steps:

1. The amount of the work done is measured as of the date of construction monitoring.

2. The existing plan is adjusted for the remaining part of the schedule.

3. Statistical simulation is performed on the corrected (adjusted) schedule and then a training (learning) sample is formed.

4. Based on the calculation called the reverse propagation of error algorithm, the vector of weighting factors is then found.

5. As of the date of the next monitoring, the actual percentage of the works done is determined and, given the earlier found weights, the construction duration forecast is made.

6. If the monitoring date is not the last one, a return to the first step is done; otherwise a transition to the end of the operational management process is accomplished.

For the neural network method of forecasting to be effective, it is necessary to discover an optimal structure of the neural network: see general recommendations in the monograph [12]. Among the relevant parameters of the artificial neural network are: the training sample size, training speed and network configuration. To come up with a forecast, the utilized network should be linked with the relevant project management program used in the calendar planning of construction [13].

Entropy assessment of expeditious construction management effectiveness: For further practical application of the construction end time forecast, let's consider the task of managing the timeliness of the works' execution in the process of construction. If it turns out during the works execution monitoring that the planned organization of construction operations does not allow the construction completion within the scheduled time, this means that uncertain situations occurred in the course of construction, which called for rescheduling; hence uncertainty arising in the process of operational management, which is generally an objective property of any system, whereas its quantitative measurement is defined by the "entropy" parameter.

The entropy definition can be found in the monograph [14]: "Entropy is a fundamental property of any random system comprised of numerous elements which are characterized by ambivalence and/or behavioral contingency (eventuality). Entropy is therefore a measure of chaos, i.e. a degree of disorder in the system and its conduct." The monograph authors [14] further arrive at two conclusions: "The lesser the system entropy is, the more stability in this system's activities exists. And vice versa: the higher the system's entropy, the messier this system is."

Entropy, as the indicator of the degree of the process uncertainty, possesses an important property. For thermodynamic entropy, this property is direct linkage of entropy with the system energy. The information entropy formally depends only on the volume of the system's information flows which in construction are formed by the organizers and performers of technological processes. Yet formation of these information flows is also directly related to managerial energy whose costs are covered in accordance with the established procedure. In an ideal case growing investment of managerial energy must minimize the rising entropy of construction operations. 
It is shown in the work [15] that entropy increments are indicative of the need to engage such extra managerial energy for organizing the construction operations, which would exclude the untimeliness of the works' execution that was the case in the past. The untimely execution of certain works is defined as deviation of actual start times and duration of works from the planned schedule. In the upshot, the average entropy of the executed timetable of the works can be expressed in the following formula:

$$
\Delta S=\frac{-\sum_{i=1}^{n} t_{i} \log _{2}\left(\sqrt{\frac{t_{i}^{2}+\tau_{i}^{2}}{\left(t_{i}+\Delta t_{i}\right)^{2}+\left(\tau_{i}+\Delta \tau_{i}\right)^{2}}}\right)}{\sum_{i=1}^{n} t_{i}},
$$

where $t_{i}$ - planned duration of the $i$-work;

$\Delta t_{i}-$ actual shifting in the duration of the $i$-work;

$\tau_{i}$ - planned or scheduled time of the $i$-work;

$\Delta \tau_{i}$-actual shifting in the beginning of the $i$-work;

$n$ - total number of works in the schedule.

Without the loss of generality, it can be surmised that for the scheduled construction operations to be executed, some extra managerial energy is required, which can be conditionally assumed to be $\Delta E_{\text {plan }}=100 \%$, while a corresponding increase in entropy will be $\Delta S_{\text {plan }}=0$. On the other hand, it can be conventionally assumed that under the pessimistic scenario of the works schedule execution, managerial energy will be reduced to zero $\left(E_{\text {pess }}=0 \%\right.$ ), whereas a corresponding increase in entropy calculated using the formula (3) will be $\Delta S_{\text {pess }}>0$. Any interim state of the construction organization will be characterized by an updated schedule of works, for which, in a general case, the calculated increase in entropy will be defined by the inequality $\Delta S_{\text {act }}<\Delta S_{\text {pess. }}$. Further, using the fact that the increment entropy is proportionate to the decrease of managerial energy, one can calculate the index of managerial energy intensification $k$ :

$$
k=\frac{\Delta S_{\text {pess }}}{\Delta S_{\text {pess }}-\Delta S_{a c t}}
$$

Index (4) calculated using this formula shows the number of times the managerial energy has to be increased for maximum compensation of the accrued untimeliness of the works done. When everything proceeds as planned, $\Delta S_{a c t}=0, k=1$ and in this case there is no need in intensifying the management energy. However, if $\Delta S_{a c t} \rightarrow \Delta S_{\text {pess }}$, managerial energy is to be intensified.

\section{Results}

Raising the effectiveness of expeditious construction management. Let's look at possible ways of managerial energy intensification, we already addressed in this article [16]. For line managers directly in charge of construction organization and administration, specific items of overhead expenses can be targeted, if their execution will affect the timeliness of construction operations and processes.

1.Expenses on line personnel wages: senior performers of works (foremen), performers of works, building site headmen, site mechanics and workers in charge of janitorial services and upkeep. 
2.Expenses on consulting and other similar services.

3.Expenses on personnel training and retraining.

4.Expenses on occupational safety.

5.Amortization and expenses on production stock repair and the fixing of tools used in construction works.

6.Expenses on the planning and design of works.

7.Expenses on the maintenance of production labs.

8.Expenses on the improvement and upkeep of construction sites.

This list shows that with expenditures on the given items of overheads growing, managerial energy can be further intensified. Thus, the item related to higher expenses on the wages of line personnel allows the intensification of managerial energy due to the introduction of additional positions. Another item related to occupational safety expenses does not intensify the managerial energy directly, though indirect impact of occupational safety upon the timeliness of the works' execution cannot be denied. Reviewing such examples, one can notice that in construction organization managerial energy is divided into loose and covert components. This fact is well-known in thermodynamic systems [14].

The practice of construction organization is such that for certain objects the above-listed items of overheads may change both due to the elimination of some items and due to the addition of other items. So it is expedient to refer to the specific lists of items, influencing the management energy called to assure the timeliness of the works' execution, as managerial energy sources. The nature of impact of each source upon the managed object is non-uniform and depends on its content. Therefore, the general additional intensity must be distributed with reference to some homogeneous indicator, such as monetary costs which is a common indicator worldwide.

To solve the task of distributing the expenses on expeditious management of the timeliness of the works' execution, we've developed a flowchart shown in Fig. 1, where stepwise execution of construction works is demonstrated. Following the works' execution at the previous stage, whose index is designated with $l$ letter, based on a review of scheduled and actual works execution times, their untimeliness is found using an integral characteristic which is assumed to be entropy growth $\Delta S_{l}$.

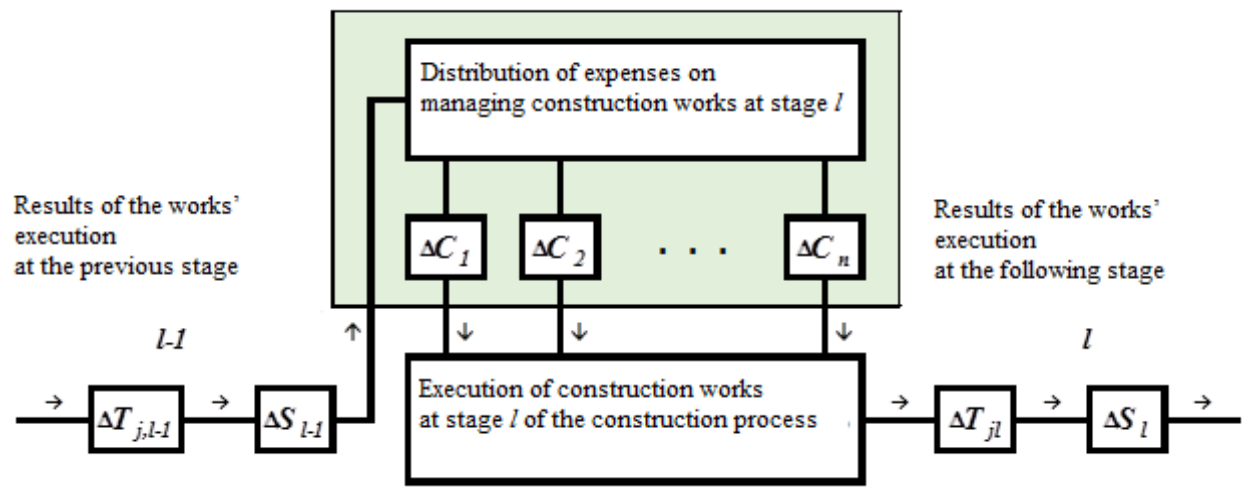

Fig. 1. Model of optimized distribution of expenses on expeditious management of the works' execution timeliness

If the managerial energy intensification index calculated using formula (4) proves equal to 1.25 , then it is necessary to increase management expenses by $25 \%$. Thus, calculated amount of extra expenses should be deemed to be the cap, whereas a specific sum of increase in the operational management expenses and its distribution between management energy sources is to be decided at the administrative level. We believe that the given 
distribution task does not lend itself easily to formalization and so its optimizing solution needs to be complemented with respective expert evaluation methods.

Let's pass on to describing the optimization setting of the distribution problem that we developed. We can represent a quantitative distribution of expenses on the earlier defined sources of managerial energy as the following formula:

$$
C=\sum_{i=1}^{n} C_{i},
$$

where $C_{i}$ - existing expenses on specified sources of managerial energy, designated by the ordering index $i$,

$C$ - total expenses on managerial energy sources, with their aggregate number designated as letter $n$.

We'd specify the absolute sum of additional expenditures related to the decrease in the untimeliness of the works as the value directly proportionate to the sum total of expenses on earmarked sources:

$$
\Delta C=(k-1) C
$$

Taking the formula (6) into account, we describe the distribution of the amount of extra expenditures on certain sources of managerial energy:

$$
\Delta C=\sum_{i=1}^{n} \Delta C_{i}
$$

Let's assume that additional investments for a concrete source of managerial energy are proportionate to the product of its original relative share in total spending by the weighting factor $w_{i}$, which must be determined via the expert evaluation procedure:

$$
\Delta C_{i}=\frac{C_{i}}{C} w_{i} .
$$

The presented formulae (5-8) define the essence of the model of distributing additional sources of managerial energy in forming the regulatory impact directed at the timely execution of construction works for the effective organization of construction operations.

\section{Conclusion}

The model of improved forecasting of the construction duration presented in this paper, developed on the basis of using neural methodology and assessment of the entropy of the relevant construction schedule, combined with optimization of the process of operational regulation of construction, will ultimately allow us to significantly increase the organizational and technological reliability of construction. 


\section{References}

1. L. I. Goncharenko, A. V. Zuikov, G. N. Semenova Journal of Advanced Research in Law and Economics, 8(5) (2017)

2. D. Radushinsky, A. Mottaeva, L. Andreeva, G. Dyakova IOP Conf. Series: Earth and Environmental Science, 90, 012137 (2017) doi :10.1088/1755$1315 / 90 / 1 / 012218$

3. L. G. Dikman, D. L. Dikman, Organization of Construction in the United States, Educational edition, p. 376 (Moscow, Publishing House for the Association of Civil Engineering Universities, 2004)

4. www.setlcity.ru (Last accessed 11.01.2019)

5. V. A. Pukhkal, A. B. Mottaeva, Magazine of Civil Engineering, 81(5), 203-212 (2018) doi: 10.18720/MCE.81.20

6. S. A. Barkalov, D. I. Golenko-Ginzburg, I. F. Nabiullin, E. A. Sidorenko, Herald of Voronezh State Technical University, 3, 38-42 (2010)

7. S. Bolotin, A. Birjukov, World Applied Sciences Journal 23, Problems of Architecture and Construction, 01-04 90001 (2013) DOI:10.5829/idosi.wasj.2013.23.pac

8. A. I. Orlov, Organizational-Economic Modeling, Part 2. Expert Estimations, Textbook (Moscow, N. E. Bauman MGTU Publishing House, 2011)

9. I. Potekhin, V. Mischenko, A. Mottaeva, A. Zheltenkov, E3S Web of Conferences, 33, 03020 (2018) doi: 10.1051/e3sconf/20183303020

10. I. Flood., P. Christophilos, Automation in Construction, 4-4, 307-320 (1996)

11. R. Kallan. Main Concepts of Neural Networks: translated from English (Moscow, PH Williams, 2001)

12. J. Patterson, A. Gibson, Deep Learning a Practitioner's Approach (O'Reilly, 2017)

13. S. A. Bolotin, A. H. Dadar, A. R. Malsagov, Herald of Civil Engineers, 3, 59-63 (2016)

14. O. L. Korolev, Using Entropy to Simulate the Processes of Decision-Making in Economics. Monograph, ed. Associate Professor A.V. Sigal. 148 p. (Simferopol, ODZHAK PH, 2013)

15. S. A. Bolotin, A. H. Dadar, R. A. Magamadov, Herald of Civil Engineers, 3, 123129 (2016)

16. S. A. Bolotin, A. H. Dadar, R. A. Magamadov, International science-technology magazine Real Estate: Economics, Management, 1, 51-56 (2017) 